\title{
Correlations in random Apollonian network
}

\author{
Zhongzhi Zhang Shuigeng Zhou* \\ Department of Computer Science and Engineering, \\ Fudan University, Shanghai 200433, China \\ Shanghai Key Lab of Intelligent Information Processing, \\ Fudan University, Shanghai 200433, China
}

\begin{abstract}
In this paper, by both simulations and theoretical predictions we study two and three node (or degree) correlations in random Apollonian network (RAN), which have small-world and scale-free topologies. Using the rate equation approach under the assumption of continuous degree, we first give the analytical solution for two node correlations, expressed by average nearest-neighbor degree (ANND). Then, we revisit the degree distribution of RAN using rate equation method and get the exact connection distribution, based on which we derive a more accurate result for mean clustering coefficient as an average quantity of three degree correlations than the one previously reported. Analytical results reveal that ANND has no correlations with respect to degree, while clustering coefficient is dependent on degree, showing a power-law behavior as $C(k) \sim k^{-1}$. The obtained expressions are successfully contrasted with extensive numerical simulations.
\end{abstract}

Key words: Complex networks, Correlations, Scale-free networks, Small-world networks, Networks

\section{Introduction}

The last few years have witnessed the birth of a new movement of interest and research in the study of complex networks as an interdisciplinary subject $[1,2,3,4,5]$. This flurry of activity, triggered by two ground-breaking papers by Watts and Strogatz on small-world networks [6] and Barabási and Albert on

* Corresponding author.

Email addresses: zhangzz@fudan.edu.cn (Zhongzhi Zhang), sgzhou@fudan.edu.cn (Shuigeng Zhou).

Preprint submitted to Elsevier Science 
scale-free networks [7], has been certainly induced by the increased computing powers and by the possibility to study the properties of a plenty of large databases of real-life networks, such as Internet, World Wide Web, metabolic networks, protein networks in the cell, co-author networks and citation networks. These networks have been identified and analyzed in the literature, the emphasis being mostly on the three basic topological characteristics, such as power-law degree distribution, small average path length (APL) and high clustering coefficient $[1,2,3,4,5]$.

However, the above mentioned three properties do not provide sufficient characterizations of the real-world systems. In fact, it has been observed that real networks exhibit ubiquitous degree correlations among their nodes $[8,9,10,11,12]$. This translates in the observation that the degrees of nearest neighbor nodes are not statistically independent but mutually correlated in most real-life networks. Correlations play an important role in the characterization of network topology, and have led to a first classification of complex networks [11]. They are thus a very relevant issue, especially in view of the important consequences that they can have on dynamical processes taking place on networks $[13,14,15,16]$.

Recently, based on the well-known Apollonian packing, deterministic Apollonian network (DAN) [17] and random Apollonian network (RAN) [18], called jointly Apollonian networks (ANs) were proposed, which have been generalized to high dimension $[19,20,21,22]$. ANs are simultaneously scale-free, smallworld, Euclidean (i.e. it can be embedded in an Euclidean lattice [17]), space filling, and with matching graphs (that is to say, in the infinite network size limit, the edges do not only completely cover the space like a Peano curve, but also never cross each other). They may provide valuable insight into the real-life networks, e.g. the maximal planarity [18] of ANs is of particular practicability for the layout of printed circuits. Moreover, ANs describe force chains in polydisperse granular packings and could also be applied to the geometry of fully fragmented porous media, hierarchical road systems, and area-covering electrical supply networks [17]. Particularly, DAN can be helpful to understand the energy landscape networks $[19,23,24]$. Very recently, ANs have attracted increasing interest from the scientific community $[25,26,27,28,29,30]$, some interesting dynamical processes, such as percolation, epidemic spreading, synchronization and random walks taking place on RAN have been investigated.

In this paper we investigate the correlation properties of nodes' connectivity of random Apollonian network (RAN) [18,21,22]. Combining a rate equation [31] in the continuous degree approximation and the boundary condition [32] of this rate equation, we work out analytically the two node correlations in RAN, measured by the average nearest-neighbor degree (ANND) of nodes with degree $k$. Then, using the genuine discrete degree distribution, we obtain the exact analytical result of mean clustering coefficient, which is the average 
value of three node correlations. Both the analytical expressions are in very good agreement with numerical simulations.

\section{Random Apollonian network}

The random Apollonian network (RAN) [18] is the stochastic version of the deterministic Apollonian network (DAN) [17]. The construction of DAN begins with $(t=0)$ a triangle and at each time step a new node is connected to the nodes of every existing triangles, omitting those triangles that had already been updated in previous steps. In the random version the triangles to be updated are selected at random, one at a time. We call the triangles to be updated active triangles.

Here we focus on the random Apollonian network (RAN). Some properties of the RAN have been investigated $[18,21,22]$. The average degree of all its nodes equals 6 . It has a power-law degree distribution $P(k) \sim k^{-3}$, and since each new node induces the addition of three triangles, one expects that the RAN has a finite clustering coefficient. It has been proved that the increasing tendency of average path length of RAN is a little slower than the logarithm of the number of nodes in RAN.

For the sake of the following investigation on correlations in RAN, we first revisit the degree distribution of RAN. Since the network size is incremented by one with each time step, we use the step value $t$ to represent a node created

at this step. Note that at time $t$ there are $2 t+1$ active triangles in RAN, and the number of active triangles containing a node with degree $k$ is also equal to $k$. Let $N_{k}(t)$ denote the average number of nodes with degree $k$ at time $t$. When a new node enters the network, $N_{k}(t)$ changes as [33]

$$
\frac{d N_{k}}{d t}=\frac{(k-1) N_{k-1}(t)-k N_{k}(t)}{2 t+1}+\delta_{k, 3}
$$

In the asymptotic limit $N_{k}(t)=t P(k)$, where $P(k)$ is the degree distribution. Eq. (1) leads to the following recursive equation

$$
P(k)= \begin{cases}\frac{k-1}{k+2} P(k-1) & \text { for } k \geq 3+1 \\ \frac{2}{5} & \text { for } k=3,\end{cases}
$$

giving

$$
P(k)=\frac{24}{k(k+1)(k+2)} .
$$

In the limit of large $k, P(k) \sim k^{-3}$ [18,21], which has the same degree exponent as the BA model [7] and the hierarchical lattice [34,35]. This obtained degree 
distribution is a more accurate result than the previous one $[18,21]$.

Notice that the growing precess of RAN actually contains the preferential attachment mechanism, which arises in it not because of some special rule including a function of degree as in Ref. [7] but naturally. Indeed, the probability that a new node created at time $t$ will be connected to an existing node $i$ is clearly proportional to the number of active triangles containing $i$, i.e. to its degree $k_{i}(t)$. Thus a node $i$ is selected with the usual preferential attachment probability $\Pi_{i}\left[k_{i}(t)\right]=k_{i}(t) /(2 t+1) \sim k_{i}(t) / 2 t$ (for large $t$ ). Consequently, $k_{i}$ satisfies the dynamical equation [1]

$$
\frac{\partial k_{i}(t)}{\partial t}=\frac{k_{i}(t)}{2 t}
$$

Considering the initial condition $k_{i}(i)=3$, we have [21]

$$
k_{i}(t)=3\left(\frac{t}{i}\right)^{1 / 2}
$$

Equation (5) shows that the degree of all nodes evolves the same way, following a power law as in the well-known BA networks [7].

\section{Correlations in Random Apollonian network}

Having obtained the exact degree distribution of the random Apollonian networks (RAN), we now study the two and three node correlations in RAN, which are merely two specific types of correlations. We ignore the long-range and multinode correlations, since the empirical data and theoretic research on such correlations is also absent.

\subsection{Two node correlations}

Two node correlations in a network can be conveniently measured by means of the quantity, called average nearest-neighbor degree (ANND), which is a function of node degree, and is more convenient and practical in characterizing degree-degree correlations. The ANND is defined by [9]

$$
k_{n n}(k)=\sum_{k^{\prime}} k^{\prime} P\left(k^{\prime} \mid k\right)
$$

If there are no two degree correlations, $k_{n n}(k)$ is independent of $k$. When $k_{\mathrm{nn}}(k)$ increases (or decreases) with $k$, the network is is said to be assortative (or disassortative) [11,12]. 
We can analytically compute the function value of $k_{n n}(k)$ for the RAN. Let $R_{i}(t)$ denote the sum of the degrees of the neighbors of node $i$, evaluated at time $t$. It is represented as

$$
R_{i}(t)=\sum_{j \in V(i)} k_{j}(t)
$$

where $V(i)$ corresponds to the set of neighbors of node $i$. The average degree of nearest neighbors of node $i$ at time $t, k_{n n}(i, t)$, is then given by $k_{n n}(i, t)=$ $R_{i}(t) / k_{i}(t)$. During the growth of the RAN, $R_{i}(t)$ can only increase by the addition of a new node connected either directly to $i$, or to one of the neighbors of $i$. In the first case $R_{i}(t)$ increases by 3 (the degree of the newly created node), while in the second case it increases by one unit. Therefore, in the continuous $k$ approximation $[1,2,3,4,5]$, we can write down the following rate equation $[31,32]$ :

$$
\frac{d R_{i}(t)}{d t}=3 \Pi_{i}\left[k_{i}(t)\right]+\sum_{j \in(V)(i)} \Pi_{j}\left[k_{j}(t)\right]=\frac{3 k_{i}(t)}{2 t}+\frac{R_{i}(t)}{2 t}
$$

The general solution of Eq. (8) is

$$
R_{i}(t)=\Phi_{0}(i) t^{\frac{1}{2}}+\frac{9}{2}\left(\frac{t}{i}\right)^{1 / 2} \ln t
$$

where $\Phi_{0}(i)$ is determined by the boundary condition $R_{i}(i)$. To obtain the boundary condition $R_{i}(i)$, we observe that at time $i$, the new node $i$ is connected to an existing node $j$ of degree $k_{j}(i)$ with probability $\Pi_{j}\left[k_{j}(i)\right]$, and that the degree of this node increase by one unit in the process. Thus,

$$
R_{i}(i)=\sum_{j=1}^{i} \Pi_{j}\left[k_{j}(i)\right]\left[k_{j}(i)+1\right]
$$

Inserting $\Pi_{j}\left[k_{j}(i)\right]=\frac{k_{j}(i)}{2 i}$ and $k_{j}(i)=3\left(\frac{i}{j}\right)^{1 / 2}$ into $R_{i}(i)$ leads to

$$
R_{i}(i)=3+\frac{9}{2} \sum_{j=1}^{i} \frac{1}{j} \simeq 3+\frac{9}{2} \ln i .
$$

So, in the large $i$ limit, $R_{i}(i)$ is dominated by the second term, yielding

$$
R_{i}(i)=\frac{9}{2} \ln i
$$

From here, we have

$$
R_{i}(t) \simeq \frac{9}{2}\left(\frac{t}{i}\right)^{1 / 2} \ln t
$$



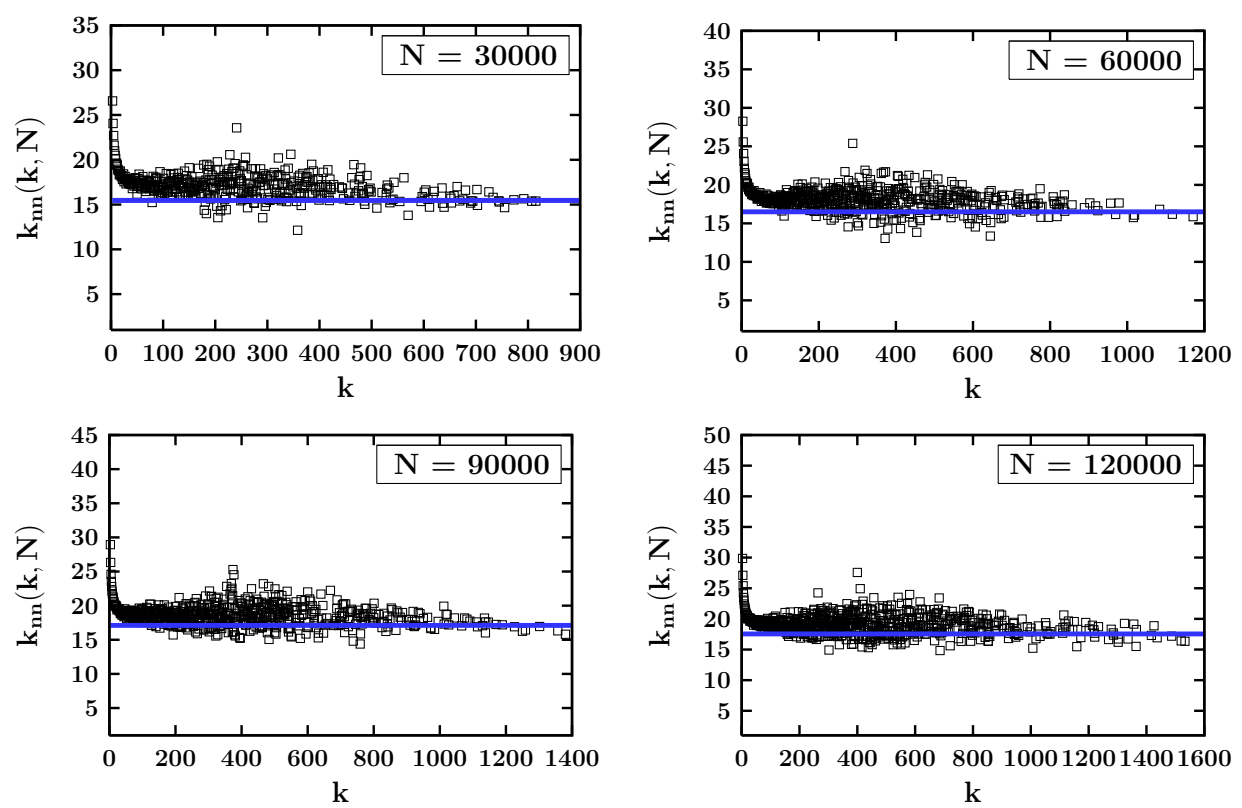

Fig. 1. Plot of average nearest-neighbor degree of the nodes with degree $k$. The squares denote the simulation results for various network sizes, while the solid lines are the theoretical result provided by Eq. (14).

and finally

$$
k_{n n}(k, t) \simeq \frac{3}{2} \ln t
$$

So, two node correlations do not depend on the degree. The ANND grows with the network size $N=t$ as $\ln t$, in the same way as in the BA model [10].

In order to confirm the validity of the obtained analytical prediction of ANND, we performed extensive numerical simulations of the RAN (see Fig. 1). To reduce the effect of fluctuation on simulation results, the simulation results are average over fifty network realizations. From Fig. 1 we observe that for large $k$ the ANND of numerical and analytical results are in agreement with each other, while the simulated results of ANND of small $k$ have a very weak dependence on $k$, which is similar to the phenomena observed in the BA model [10]. This $k$ dependence, for small degree, cannot be detected by rate equation approach, since it has been formulated in the continuous degree $k$ approximation.

From Eq. (14), we can also easily know that the evolution of ANND of all nodes is the same, showing a weak logarithmic divergence with evolution time (network size). To confirm this analytical prediction we also perform numerical simulations. In Fig. 2 we report the numerical results, which are in good agreement with this prediction provided by Eq. (14). 


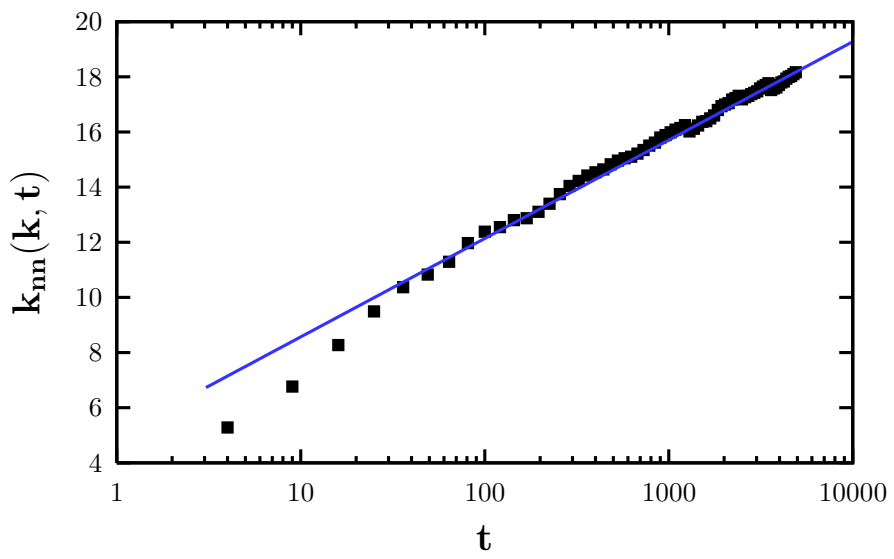

Fig. 2. Semilogarithmic graph of time evolution for the ANND of the node added to the system at time 2 . The straight line follows $k_{n n}(k, t)=\frac{3}{2} \ln t$ as predicted by Eq. (14). All data are from the average of 50 independent runs.

\subsection{Three node correlations}

Three node correlations can be measured by means of the conditional probability $P\left(k^{\prime}, k^{\prime \prime} \mid k\right)$ that a node of degree $k$ is simultaneously connected to nodes with degree $k^{\prime}$ and $k^{\prime \prime}$. The direct evaluation of the $P\left(k^{\prime}, k^{\prime \prime} \mid k\right)$ is generally difficult. To overcome this problem, another interesting alternative quantity, i.e. clustering coefficient, is frequently used. By definition, the clustering coefficient [6] of nodes with degree $k, C(k)$, is defined as the probability that two neighbors of a node with degree $k$ are also neighbors themselves. The average clustering coefficient of the whole network is then given as

$$
C=\sum_{k} P(k) C(k)
$$

Now we analytically estimate the average clustering coefficient $C$ of RAN by means of the clustering spectrum $C(k)$. In the RAN, for a node of degree $k$, the exact value of its clustering coefficient is $[18,21,22]$

$$
C(k)=\frac{4 k-6}{k(k-1)},
$$

which depends on degree $k$. For large $k$, the degree-dependent clustering $C(k)$ is inversely proportional to degree $k$, the same behavior has been analytically found in some growing network models such as deterministic (random) pseudofractal scale-free networks and their variants $[36,37,38,39,40,41,42]$, as well as some real systems [43].

Using Eq. (15), the average clustering coefficient $C$ of RAN can be easily obtained as the mean value of $C(k)$ with respect to the degree distribution $P(k)$ expressed by Eq. (3). The result is 


$$
\begin{aligned}
C & =\sum_{k=3}^{\infty} P(k) C(k)=\sum_{k=3}^{\infty} \frac{24}{k(k+1)(k+2)} \frac{4 k-6}{k(k-1)} \\
& =\sum_{k=3}^{\infty} \frac{24}{k(k+1)(k+2)}\left(\frac{4}{k-1}-\frac{6}{k(k-1)}\right)=C_{1}-C_{2},
\end{aligned}
$$

where $C_{1}=\sum_{k=3}^{\infty} \frac{24}{k(k+1)(k+2)} \frac{4}{k-1}$ and $C_{2}=\sum_{k=3}^{\infty} \frac{24}{k(k+1)(k+2)} \frac{6}{k(k-1)}$. We now compute in detail $C_{1}$ and $C_{2}$, respectively. First, we can decompose $C_{1}$ into the sum of four terms as

$$
C_{1}=\sum_{k=3}^{\infty}\left(\frac{16}{k-1}-\frac{48}{k}+\frac{48}{k+1}-\frac{16}{k+2}\right)=\frac{4}{3}
$$

Analogously to Eq. (18), we get

$$
C_{2}=\sum_{k=3}^{\infty}\left(\frac{24}{k-1}+\frac{36}{k}-\frac{72}{k^{2}}+\frac{72}{k+1}-\frac{12}{k+2}\right)=119-12 \pi^{2}
$$

where we have used the fact that $\sum_{m=1}^{\infty} \frac{1}{m^{2}}=\frac{1}{6 \pi^{2}}$. Substituting Eqs. (18) and (19) into Eq. (17), we have

$$
C=\frac{4}{3}-\left(119-12 \pi^{2}\right) \simeq 0.768
$$

Thus the average clustering coefficient $C$ of RAN is large and independent of network size. Since Eqs. (3), (16), (18), and (19) are exact, it is the same with the obtained $C$ value. We have performed extensive numerical simulations of the RAN. In Fig. 3, we present the simulation results about the average clustering coefficient of RAN, which are in complete agreement with the analytical value.

It should be mentioned that previous jobs [18,21] have studied the average clustering coefficient of RAN, using continuum approximation and integral methods, and have acquired a analytical value of $C=\frac{46}{3}-36 \ln \frac{3}{2} \simeq 0.737$. From Fig. 3, we can see that there is a difference between this analytical value and simulated ones. Where does the difference come from? And why Eq. (20) is more accurate? These might be explained as follows. In RAN, a node may have only an integer number of connections (degrees). The continuum approach used previously $[18,21]$ is under the assumption that the degree distribution $P(k)$ is modeled by the continuous distribution $P_{c}(k)=\alpha k^{-3}(k \geq$ $3)$, where $\alpha=18$ is a normalization constant. It does not properly account for the fraction (i.e. degree distribution) of nodes with small degrees [44], and therefore has a discrepancy with the exact result for RAN with the genuine discrete degree distribution $P_{d}(k)$ expressed by Eq. (3) [see Fig. 3]. However, 


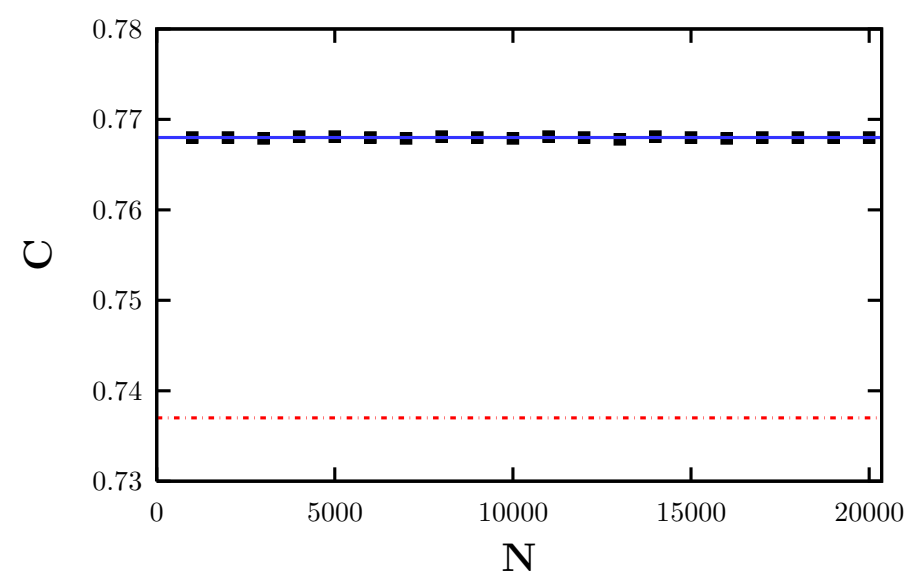

Fig. 3. Average clustering coefficient $C$ of RAN vs the network size $N$. The solid line shows the exact result $\frac{4}{3}-\left(119-12 \pi^{2}\right)$, while the dashed line depicts the previous analytic value $\frac{46}{3}-36 \ln \frac{3}{2}$. The squares denote the results of simulations. Each data is obtained by fifty independent network realizations.

it is worth noticing that (a) $P_{c}(k)$ and $P_{d}(k)$ are equal, asymptotically, in the limit of large $k$ and (b) the difference is most pronounced for $k \approx 3$. The discrepancy is mainly due to the values of $P(k)$ for small $k$. Thus, the discrete method, omitting a continuum assumption, seems more suitable for obtaining exact results for some challenging tasks [1].

\section{Conclusion}

In this article, we have done an analytical study of the correlations between the degrees of neighboring nodes in random Apollonian networks (RAN). Applying rate equation method in the continuous $k$ approximation, together with its boundary condition that node $i$ was added to the system at time $i$ with the expected sum of degrees of its neighbors being $R_{i}(i)=3+\frac{9}{2} \ln i$, we have provided the solution of the average degree of nearest neighbors as the measure of two degree correlations. The obtained result shows that RAN lacks two degree correlations, which means that the ANND of nodes with degree $k$ is independent of $k$. Moreover, we have obtained the exact degree distribution of RAN, on the basis of which we have attained the exact value of mean clustering coefficient as an average quantity of three node correlations. We found that RAN are highly clustered, and the clustering spectrum scales as $C(k) \sim k^{-1}$, exhibiting hierarchical organization and modularity. These obtained structure properties may be helpful to understand and explain the workings of systems built on RAN. Many physical processes, such as disease spread and random walks, taking place in RAN exhibit different behaviors than those in the classic BA networks, which are relevant to these particular topologies of RAN $[18,26,30]$. 


\section{Acknowledgment}

This research was supported by the National Natural Science Foundation of China under Grant Nos. 60373019, 60573183, and 90612007, and the Postdoctoral Science Foundation of China under Grant No. 20060400162. The authors thank Zhen Shen for his assistance in preparing the manuscript.

\section{References}

[1] R. Albert and A.-L. Barabási, Rev. Mod. Phys. 74, 47 (2002).

[2] S. N. Dorogovtsev and J. F. F. Mendes, Evolution of Networks: From Biological Nets to the Internet and $W W W$ (Oxford University Press, New York, 2003).

[3] R. Pastor-Satorras and A. Vespignani, Evolution and Structure of the Internet: A Statistical Physics Approach (Cambridge University Press, Cambridge, England, 2004).

[4] M. Newman, A.-L. Barabási, D. J. Watts, The Structure and Dynamics of Networks (Princeton University Press, Princeton, 2006).

[5] S. Boccaletti, V. Latora, Y. Moreno, M. Chavez and D.-U. Hwanga, Phys. Rep. 424, 175 (2006).

[6] D.J. Watts and H. Strogatz, Nature (London) 393, 440 (1998).

[7] A.-L. Barabási and R. Albert, Science 286, 509 (1999).

[8] S. Maslov and K. Sneppen, Science 296, 910(2002)

[9] R. Pastor-Satorras, A. Vázquez and A. Vespignani, Phys. Rev. Lett. 87, 258701 (2001).

[10] A. Vázquez, R. Pastor-Satorras and A. Vespignani, Phys. Rev. E 65, 066130 (2002).

[11] M. E. J. Newman, Phys. Rev. Lett. 89, 208701 (2002).

[12] M. E. J. Newman, Phys. Rev. E 67, 026126 (2003).

[13] M. Boguñá and R. Pastor-Satorras, Phys. Rev. E 66, 047104 (2002).

[14] Y. Moreno and A. Vázquez, Eur. Phys. J. B 31, 265 (2003).

[15] A. Vázquez and Y. Moreno, Phys. Rev. E 67, 015101(R) (2003).

[16] P. Echenique, J. Gómev-Gardeñes, Y. Moreno, and A. Vázquez, Phys. Rev. E 71, 035102(R) (2003).

[17] J.S. Andrade Jr., H.J. Herrmann, R.F.S. Andrade and L.R.da Silva, Phys. Rev. Lett. 94, 018702 (2005). 
[18] T. Zhou, G. Yan, and B.H. Wang, Phys. Rev. E 71, 046141 (2005).

[19] J.P.K. Doye and C.P. Massen. Phys. Rev. E 71, 016128 (2005).

[20] Z.Z. Zhang, F. Comellas, G. Fertin and L.L. Rong, J. Phys. A 39, 1811 (2006).

[21] Z.Z. Zhang, L.L. Rong and F. Comellas, Physica A 364, 610 (2006).

[22] Z.Z. Zhang, L.L. Rong and S.G. Zhou, Phys. Rev. E 74, 046105 (2006).

[23] J.P.K. Doye. Phys. Rev. Lett. 88, 238701 (2002).

[24] J.P.K. Doye and C.P. Massen. J. Chem. Phys. 122, 084105 (2005).

[25] R.F.S. Andrade and H.J. Herrmann, Phys. Rev. E 71, 056131 (2005).

[26] P. G. Lind, J.A.C. Gallas, and H.J. Herrmann, Phys. Rev. E 70, 056207 (2004).

[27] R.F.S. Andrade, J.G.V. Miranda, Physica A 356, 1 (2005).

[28] Y. Hayashi and J. Matsukubo, Lecture Notes in Computer Science 3993, 1106 (2006).

[29] Y. Hayashi and J. Matsukubo, Phys. Rev. E 73, 066113 (2006).

[30] Z.-G. Huang, X.-J. Xu, Z.-X. Wu, and Y.-H. Wang, Eur. Phys. J. B 51, 549 (2006).

[31] G. Szabó, M. Alava, and J. Kertész, Phys. Rev. E 67, 056102 (2003).

[32] A. Barrat and R. Pastor-Satorras, Phys. Rev. E 71, 036127 (2005).

[33] P.L. Krapivsky, S. Redner, and F. Leyvraz, Phys. Rev. Lett. 85, 4629 (2000).

[34] A.N. Berker and S. Ostlund, J. Phys. C 12, 4961 (1979).

[35] M. Hinczewski and A. N. Berker, Phys. Rev. E 73, 066126 (2006).

[36] S.N. Dorogovtsev, A.V. Goltsev, and J.F.F. Mendes, Phys. Rev. E 65, 066122 (2002).

[37] F. Comellas, G. Fertin and A. Raspaud, Phys. Rev. E 69, 037104 (2004).

[38] Z. Z. Zhang, L. L. Rong, and S. G. Zhou, Physica A 377, 329 (2007).

[39] S.N. Dorogovtsev, J.F.F. Mendes, and A.N. Samukhin, Phys. Rev. E 63, 062101 (2001).

[40] J. Ozik, B.-R. Hunt, and E. Ott, Phys. Rev. E 69026108 (2004).

[41] Z.Z. Zhang, L.L. Rong and C.H. Guo, Physica A 363, 567 (2006).

[42] Z.Z. Zhang, L.L. Rong and F. Comellas, J. Phys. A 39, 3253 (2006).

[43] E. Ravasz and A.-L. Barabási, Phys. Rev. E 67, 026112 (2003).

[44] S. N. Dorogovtsev and J. F. F. Mendes, Phys. Rev. Lett. 87, 219801 (2001). 